\title{
Caracterización de los ovinocultores y sus sistemas productivos en el litoral sur del Perú
}

\author{
Characterization of sheep breeders and their productive systems in southern coast of Peru
}

Iván Salamanca Montesinos ${ }^{1}$, Nilton Gómez Urviola ${ }^{2}$, Maria Clorinda Soares Fioravanti ${ }^{1}$, Jose Robson Bezerra Sereno ${ }^{3}$

* Autor de correspondencia

\section{Resumen}

En Perú, la ovinocultura es importante para los pequeños productores, que representan el 75\% de la población rural nacional; pero existe poca información sobre esta actividad en la zona sur del país, por esa razón este estudio planteó como objetivo caracterizar los ovinocultores y sus sistemas productivos. Durante el año 2012, en el municipio de Ite, litoral de la región Tacna, se entrevistaron 32 criadores con preguntas abiertas y cerradas. Los datos colectados fueron tabulados en planillas electrónicas, para analizarlos en frecuencias absolutas y relativas, así como estadística descriptiva usando el programa computacional R. Ocho entrevistados eran pastores Aymara que manejan semiextensivamente rebaños ovinos y caprinos en los Humedales de Ite. Los otros eran agricultores, quienes producen pequeños rumiantes principalmente con el sistema de estaca en las chacras. La asistencia técnica y extensión rural son inadecuadas, afectando negativamente los rebaños, compuestos por ovinos criollos y mestizos. En Ite, la producción de ovinos es tradicional, subsistiendo porque la carne es apreciada en la región y la crianza una costumbre familiar. Finalmente se sugiere mayor apoyo gubernamental, para aplicar tecnologías que permitan una ovinocultura sustentable.

Palabras clave: Agricultores; chacras; Humedales de Ite; ovinos; pastores Aymara.

\begin{abstract}
In Peru, sheep farming is important for smallholders, which represent the $75 \%$ of national rural population; but there is little information about this activity in the southern zone of the country, by this reason this study aimed to characterize the sheep breeders and their productive systems. During the year 2012, in the municipality of Ite, coast of Tacna region, 32 breeders were interviewed with open and closed questions. The collected data were tabulated in electronic spreadsheets, to analyze in absolute and relative frequencies, as well as descriptive statistics using the software R. Eight interviewees were Aymara shepherds that handled semiextensively herds of sheep and goats in the Ite's Humedales. The others were smallholders, who produce small ruminants mainly with stake system on farms. The technical assistance and rural extension are inadequate, affecting negatively the herds, composed by creole and crossbred sheep. In Ite, sheep production is traditional, subsisting because the meat is appreciated in the region and the breeding a family custom. Finally is suggested more government support, for applying technologies that allow a sustainable sheep farming.
\end{abstract}

Keywords: Aymara shepherds; farms; Ite's Humedales; sheep; smallholders.

\section{Introducción}

En Perú, la ovinocultura tiene importancia económica igual o mayor que la bovinocultura (Flores, 2008; Aliaga, 2009) y fue introducida por los colonizadores españoles en el siglo XVI (Alencastre y Gómez, 2005), siendo adoptada en las comunidades indígenas andinas, como parte de su cultura ancestral pastoril que ya existía con la crianza de rebaños de llamas y alpacas (Fulcrand, 2005; Gade, 2013). Los ovinos proporcionan carne, lana, piel y estiércol, comercializándose informalmente el 70\% de estos subproductos, el restante es para consumo del criador (Flores et al., 2007; Díaz, 2013). El ovino se adapta a diferentes climas, desde los más calurosos hasta los más fríos (Aliaga, 2009), habitando en las tres regiones naturales peruanas, que son la Costa del océano Pacífico, la Sierra Andina y Selva Amazónica. En esos ambientes esa especie se cría de forma mixta con bovinos, caprinos y camélidos sudamericanos, mayormente en un sistema económico familiar de subsistencia (Díaz, 2007).

Según el último censo agropecuario nacional de 2012, en Perú los ovinos suman 9,5 millones, de los cuales 33,8 mil pertenecen a la región Tacna, donde el municipio rural de Ite con 3916 animales poseía el mayor rebaño. En la región Tacna destacaron los ovinos criollos (68\%), en comparación a los Hampshire Down (17\%) y Black Belly (4\%) (INEI, 2013). En Ite, la ovinocultura es una actividad 
creciente e importante para los pequeños productores, reportándose el año 2014 un número de 6113 ovinos, siendo criados 4234 en el ecosistema de los Humedales y 1879 en las chacras (MDI, 2014a).

Debido a la poca información documentada sobre la ovinocultura en la región Tacna y el número significativo de ovinos en Ite, se planteó como objetivo caracterizar en esa localidad a los ovinocultores y sus sistemas productivos, lo cual sería un aporte para la crianza de pequeños rumiantes en el sur del Perú.

\section{Material y métodos}

El año 2012, en el distrito de Ite, provincia Jorge Basadre Grohmann de la región Tacna, litoral sur del Perú (Figura 1), fueron entrevistados 32 ovinocultores, mayores de 18 años de edad e hispano hablantes, usando un cuestionario y grabador de voz digital.

La localidad se sitúa en la Latitud sur $17^{\circ} 50^{\prime} 27^{\prime \prime}$ y Longitud oeste $70^{\circ} 57^{\prime} 47^{\prime \prime}$, posee un clima templado y dos estaciones anuales marcadas, el invierno (junio a agosto), con temperatura promedio de $16^{\circ} \mathrm{C}$, entre 2,2 a $3,2 \mathrm{~mm}$ de precipitaciones y $86 \%$ de humedad; ya el verano (diciembre a febrero) registra alrededor de $28^{\circ} \mathrm{C}$, con 0,2 a $0,0 \mathrm{~mm}$ de lluvia y 66\% de humedad. (Vizcarra, 2008; MDI, 2014b). Al este del océano Pacífico existen los Humedales, que son áreas inundadas, producto de las aguas de la capa freática del municipio de Ite y las aguas del río Locumba, que desemboca en el litoral formando en su último trecho ese pantano (MINAG, 2007; Vizcarra, 2008). Este ecosistema costero está constituido por fauna y flora nativa, con 12 km de extensión y 2000 hectáreas de superficie, donde 19 familias de pastores Aymara aprovechan la vegetación para crianza semiextensiva de ovinos y caprinos (Montesinos et al., 2012).

Continuando al este, con una elevación de 175 metros sobre el nivel del mar y $848,34 \mathrm{~km}^{2}$ de superficie, se encuentra la parte rural y urbana del municipio, que en 2012 tenía una población de 3503 personas, de las cuales 1786 poseían propiedades rurales o "chacras" (MDI, 2014b), dependiendo de la actividad agropecuaria como sustento familiar. El censo municipal de 2014 registró 126 ovinocultores en este ambiente (MDI, 2014a). En Ite, tanto los Humedales como las chacras son dos lugares topográficamente diferentes, donde se desarrolla habitualmente la ovinocultura.

Las entrevistas fueron aplicadas por una sola persona, utilizando la metodología de Perezgrovas y Castro (2000), que aborda temas referentes al perfil del criador y su rebaño. Los datos obtenidos fueron transcritos a planillas electrónicas, clasificando las respuestas de los dos grupos de ovinocultores en variables cuantitativas y cualitativas, para transformarlas en frecuencias absolutas y relativas. Los valores cuantitativos absolutos se analizaron estimando promedios y desviación estándar, comparándolos mediante análisis de varianza y Test Scott Knott a nivel de significancia del 5\%, con el paquete estadístico easynova (Arnhold, 2013) del programa computacional R (R Development Core Team, 2011).

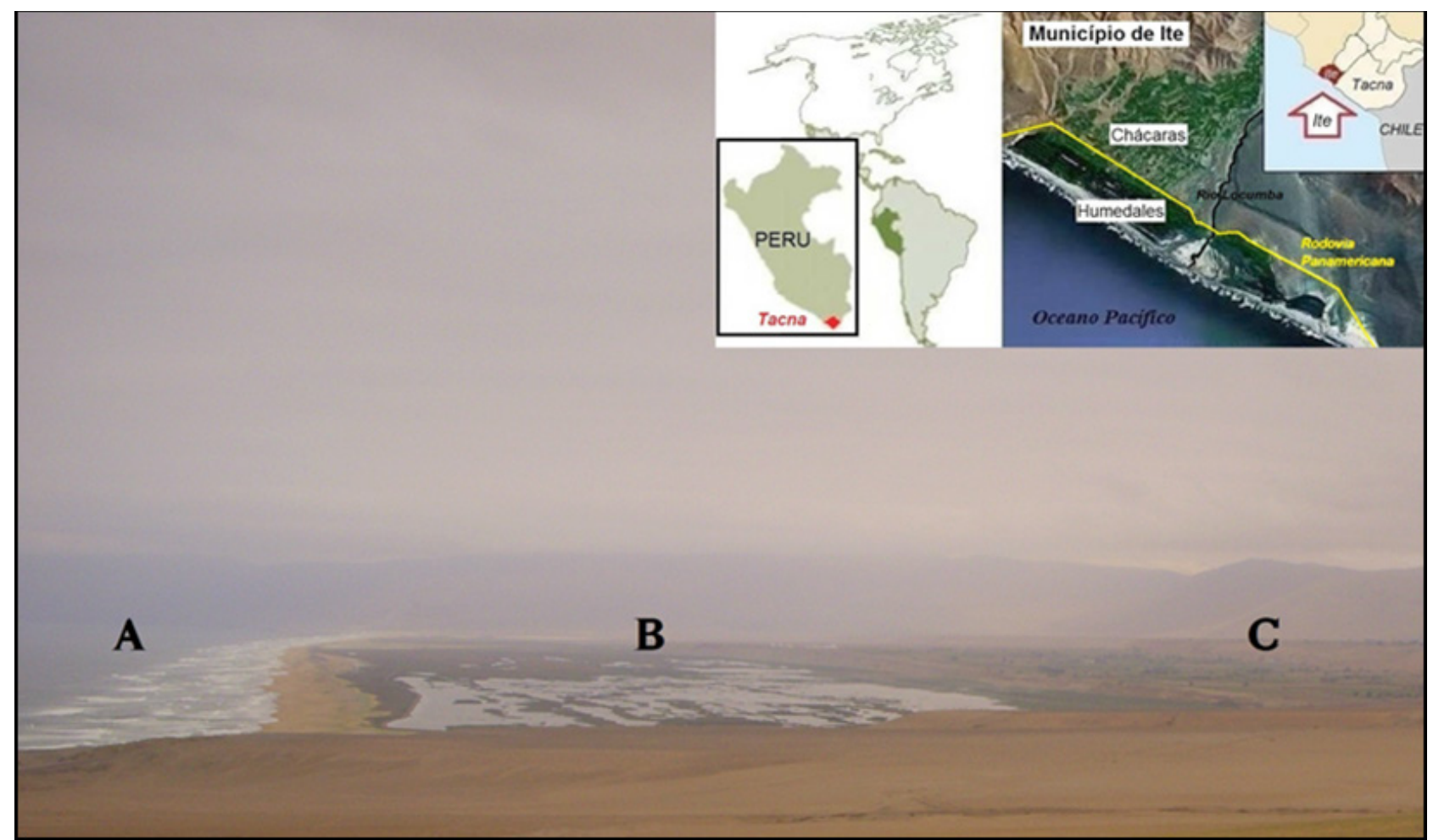

Figura 1. Municipio rural de Ite, región Tacna, litoral sur del Perú. Océano Pacífico (A) limitando con los Humedales (B) y las chacras $(C)$ 


\section{Resultados y discusión}

\section{Perfil del ovinocultor}

De los 32 ovinocultores entrevistados, ocho eran pastores de los Humedales (PASTHU) y los demás agricultores de las chacras (AGRICH), oriundos de diferentes regiones del Perú, por lo cual existe cierta heterogeneidad étnica (Figura 2).

Los PASTHU hablan su lengua nativa Aymara y el castellano. Los 24 AGRICH hablan castellano, pero algunos eran bilingües, siendo nueve Aymaras y dos Quechuas. Muchos entrevistados provienen de la sierra peruana, debido al fenómeno migratorio que ocurre en las ciudades costeras, donde llegan pobladores andinos en busca de mejores condiciones de vida, escapando de la pobreza que dejó el terrorismo en las últimas décadas (Escárzaga et al., 2002). En Perú, las familias andinas consideran al ovino como su principal fuente de carne (Gómez y Gómez, 2009), costumbre que mantienen estos residentes de Ite. Algo parecido ocurrió con productores rurales en Tabasco, sur de México, donde la identidad familiar fue influenciada por inmigrantes del centro del país, que acostumbran consumir carne ovina (Nuncio-Ochoa et al., 2001).

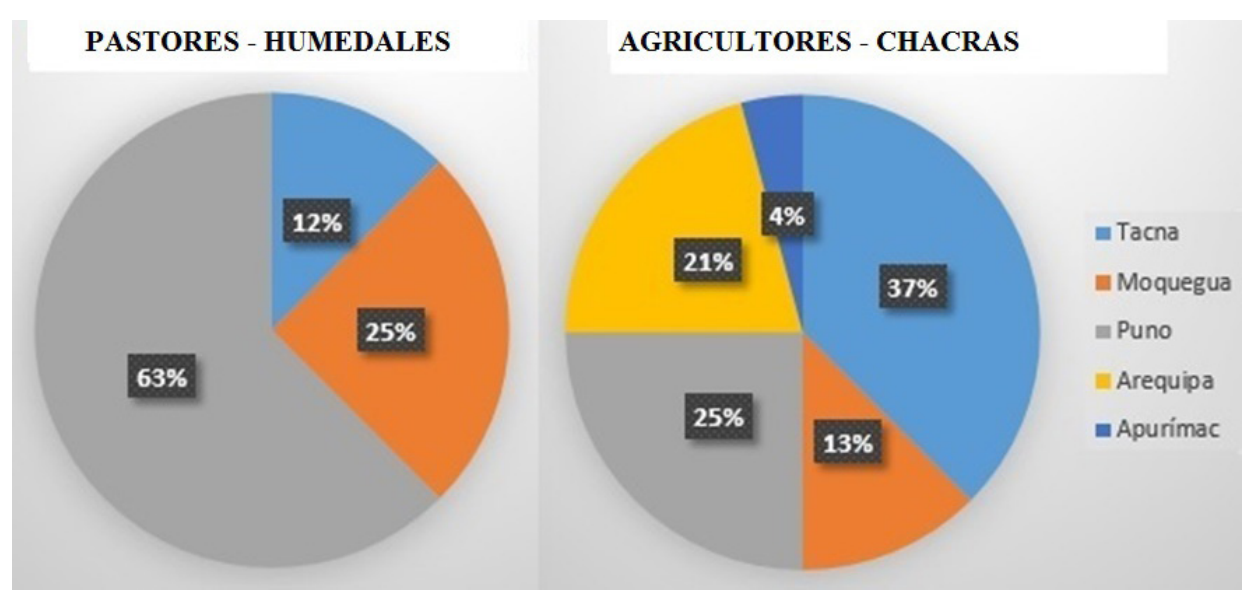

Figura 2. Regiones de origen de los ocho pastores de los Humedales y 24 agricultores de las chacras entrevistados en Ite, Perú

Perezgrovas y Castro (2000) en México; Fulcrand (2005) en Perú; Sponenberg y Taylor (2009) en Estados Unidos; Stemmer et al., (2010) en Bolivia; De la Barra et al. (2011) en Chile y De la Rosa et al. (2014) en Argentina describieron comunidades de indígenas Tzotziles, Quechuas, Navajos, Aymaras, Williches y Qom, respectivamente, que sobreviven de la crianza ovina. Lo cual demuestra la importancia de esta especie animal para la seguridad alimentaria de territorios aislados y poblaciones marginadas.

Al visitar los Humedales se encontró más mujeres como encargadas del rebaño $(62,5 \%)$, ya en las chacras fue lo contrario (37,5\%). Esto indica que las PASTHU están implicadas directamente en tareas de la actividad (Figura 3). Algo particular en la ovinocultura peruana, pues la mujer cumple un papel fundamental (Aliaga, 2009).
Las edades de los entrevistados eran en promedio 48,1 años para los PASTHU y 49,9 años para los AGRICH, próximas a las encontradas en criadores dominicanos por Valerio et al. (2010) y en españoles por Marín-Bernal y Navarro-Ríos (2014). El tiempo de experiencia como ovinocultores fue de 29 años para los PASTHU y 27,7 años para los AGRICH. La mayoría de participantes tenía más de 20 años en la actividad (PASTHU 62,5\%; AGRICH $62,4 \%$ ), algo común en los campesinos peruanos, pues desde tiempos coloniales criar ovinos es parte de su cultura pastoril, que ya existía con el manejo de llamas y alpacas (Fulcrand, 2005; Gade, 2013). Había 29,2\% de AGRICH con menos de 10 años en la actividad, criadores neófitos que optaron por el ovino debido a la demanda de su carne o el poco espacio de tierra para tener bovinos.

La escolaridad de los PASTHU era mínima, predominando el nivel primario $(62,5 \%)$ sobre el secundario $(25 \%)$ y una anciana es analfabeta. Esta situación social precaria también fue descrita por De la Rosa et al. (2014), en ovinocultores indígenas Qom del nordeste argentino. Los AGRICH tenían mayor grado de escolaridad secundaria (45,8\%), inclusive dos de ellos poseen estudios universitarios.

Al preguntar sobre medios de comunicación, todos citaron el celular, la televisión y radio, lo que indica acceso a energía eléctrica y tecnología. Los dos últimos aparatos electrónicos fueron registrados por Altafin et al. (2011), al visitar productores brasileros en Unaí (Minas Gerais), destacando la radio por ser portátil, llevándola al campo mientras trabajan, algo muy común también entre los pobladores rurales de Ite. Cabe resaltar el mayor hábito de lectura de los AGRICH (79,2\%) en comparación a los PASTHU (37,5\%), ya que acostumbran comprar periódicos de noticias regionales y nacionales. Únicamente tres AGRICH saben usar internet, pues trabajaban para el municipio en funciones que necesitan esa herramienta tecnológica.

Las familias de los PASTHU estaban compuestas en promedio por 5,8 personas y de los AGRICH por 4,8 . Cinco familias de PASTHU y 13 de AGRICH tenían niños en edad escolar, siendo que esas nuevas generaciones quizás pertenezcan a sectores sociales más desarrollados (Bedotti et al., 2005), lo que puede llevar al abandono de la ovinocultura, pues la población joven suele dejar el campo para vivir en la ciudad (Segundo et al., 2006), algo que ocurre en áreas rurales del Perú en las últimas décadas.

Los entrevistados también manifestaron tener trabajos 


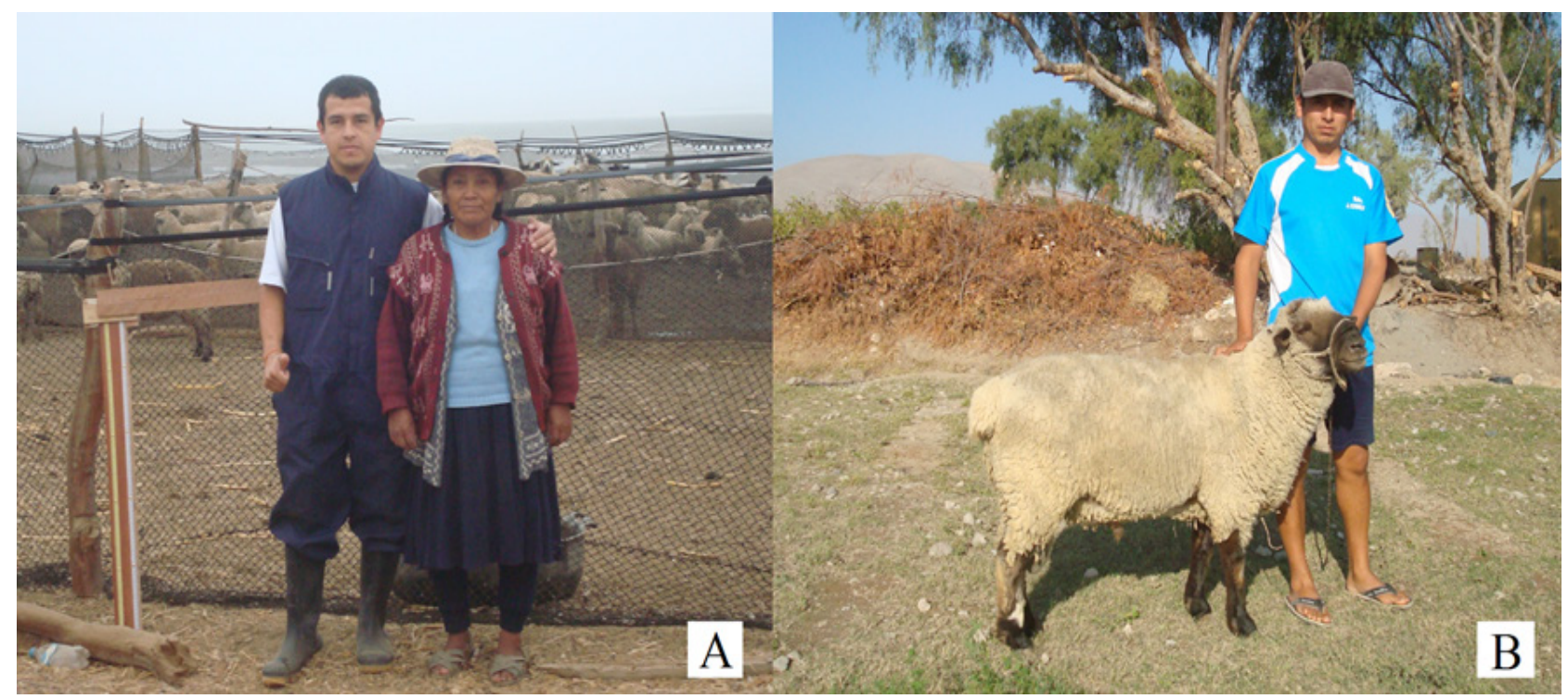

Figura 3. Pastora Aymara de los Humedales, vistiendo ropa típica de su etnia (A). Agricultor de las chacras y su carnero mestizo Hampshire Down (B)

no agrícolas (87,5\% PASTHU; 50\% AGRICH) para complementar su renta familiar, situación parecida fue observada en ovinocultores brasileros y mexicanos por Holanda Junior et al. (2004) y Nuncio-Ochoa et al. (2001). En Ite muchos ovinocultores de sexo masculino trabajaban para el municipio, algunos en funciones que requieren conocimiento técnico de oficina y la mayoría en labores que no necesitan mano de obra calificada (albañiles, vigilantes, etc.). Frente a esto delegaban la atención del rebaño a las mujeres, niños y ancianos, como también fue descrito por Fulcrand (2005) y Stemmer et al. (2010), con tareas más leves, destacando el manejo de crías, pastoreo, administración de agua y atendimiento de animales enfermos. Los hombres realizaban servicios más pesados, como corte de cola, castración, beneficio, colecta de estiércol, aplicación de vermífugos y vacunas.

Esta mano de obra familiar también fue registrada entre ovinocultores mexicanos (Martínez et al., 2009) y caprinocultores argentinos (Bedotti et al., 2005), algo típico de las producciones de subsistencia (Daskalopoulou y Petrou, 2002). De otra parte, 11 AGRICH tenían empleados fijos y/o temporales. Los trabajadores fijos atendían animales o cultivos, y los temporales eran contratados en época de cosechas, como ají, maíz o cebolla, vegetales más adaptados a este ambiente árido del litoral. En la sierra sur de España algunas explotaciones de ovinos Segureños tenían trabajadores fijos para el pastoreo y/o eventuales para las pariciones (Marín-Bernal y Navarro-Ríos, 2014).

En los Humedales, el caprino es la otra especie animal que sustenta a las familias de los pastores Aymara. Algo similar a lo encontrado en los llanos venezolanos (Morantes et al., 2008) y semiárido brasilero (Santos et al., 2012), lugares donde la ovinocaprinocultura es una importante actividad económica para la población rural. Algunos PASTHU criaban bovinos, suínos, aves, cuyes
(Cavia porcellus) o producían ají (Capsicum annuum), en menor escala y para consumo propio.

En las chacras como parte de la renta familiar destacó la bovinocultura lechera $(79,2 \%)$, ya que los agricultores poseen mayor interés en esa actividad ganadera, que necesita de gastos variados como la compra de animales y equipamientos (Nuncio-Ochoa et al., 2001). También resaltó la producción de ají $(79,2 \%)$, en su variedad amarilla, panca y páprika, para venta a compradores que llevan ese producto hasta los países fronterizos de Bolivia y Chile. En las entrevistas fueron identificados varios criadores de cuyes $(62,5 \%)$, roedores domésticos tan comunes en las propiedades como las gallinas $(62,5 \%)$, manejados básicamente por las mujeres y que representan una fuente proteica a bajo costo (Avilés et al., 2014). Algunos agricultores tenían suínos y conejos, especies también encontradas por Lopéz et al. (2013) en la ganadería de traspatio, la cual busca criando en casa la seguridad alimenticia del pequeño productor.

La mitad de los entrevistados pidieron en alguna ocasión préstamo monetario, a entidades financieras públicas y particulares, como Caja Municipal de Tacna, Mibanco y Pro Mujer, evidenciando accesibilidad al crédito rural, ya que en Perú en los últimos años ese sector creció significativamente (Santillana, 2012). Algo que también ocurre en otros países de Sudamérica, como Brasil, donde Farías et al. (2014) describieron que en el semiárido de Ceará, criadores de ovinos y caprinos tenían acceso al crédito rural, vía el Programa Nacional de Fortalecimiento de la Agricultura Familiar (PRONAF).

Todos los PASTHU entrevistados pertenecían a la asociación de criadores de pequeños rumiantes "San Juan de Ite", porque como gremio esperaban mayor apoyo del gobierno, pues individualmente eran ignorados. Solo45,8\% de los AGRICH participaba en alguna asociación, el 
restante acusaba falta de tiempo, desinformación o vejez como limitantes para su cooperativismo, confirmando que la escasa organización de los productores es una deficiencia de la ovinocultura peruana (Gonzáles, 2008). Las asociaciones de AGRICH eran de bovinocultores lecheros $(72,7 \%)$, productores de pequeños rumiantes $(18,2 \%)$, cuyes $(18,2 \%)$, ají $(18,2 \%)$ y aceituna $(9,1 \%)$, justificando esa adhesión por beneficios públicos y privados que pueden recibir para su actividad.

En términos de asistencia y calificación técnica, el año 2012 los PASTHU informaron que no las recibían desde hace dos años, cuando concluyó un proyecto caprino municipal. Esto indica que en los Humedales el atendimiento veterinario y capacitación solo es estatal, tal como informaron Bedotti et al. (2005) para varios caprinocultores del oeste pampeano argentino. La mayor parte de los AGRICH tenían asistencia técnica y charlas (62,5\%), pero destinadas a bovinos lecheros y cultivos, a través de proyectos municipales. Los AGRICH que no participaban $(37,5 \%)$ en los cursos justificaron su ausencia por falta de tiempo, transporte o desinformación.

Estas situaciones son negativas, pues la asistencia técnica al ganadero es indicador de mejores condiciones de producción (Bedotti et al., 2005) y su capacitación permitiría incorporación de nuevas tecnologías, modificando la mentalidad de tener ovejas a la de "ser criador" de ellas (Fulcrand, 2005). En muchas entrevistas se verificó que solo el padre de familia participaba de cursos, algo que debe cambiar pues las mujeres también cuidan los animales, cumpliendo un rol decisivo en la agricultura (Hernández, 2012).

Los PASTHU querían cursos sobre ovinocaprinocultura, con reuniones apenas entre criadores y días de campo visitando rebaños vecinos, para ver el manejo de otros propietarios, experiencias útiles en una actividad rentable (Holanda Junior, 2005; Santos et al., 2012). Los AGRICH se interesaron por charlas sobre bovinos lecheros y ovinos. Todos los entrevistados deseaban capacitación, generalmente mensual y en horarios de la tarde cuando acaba su jornada de campo o de mañana cuando los animales pastorean.

\section{Características del rebaño}

Los entrevistados conocían la procedencia de sus animales, como también reportaron Peña et al. (2016) en ovinocultores argentinos. La mayor parte compró sus ovinos (PASTHU 75\%; AGRICH 79,2\%) y el resto de ganaderos los heredó de la familia o ambas modalidades. Los PASTHU tenían de 100 a 300 ovinos, con un promedio de 189 por criador, siendo el tamaño de rebaño similar a los estudiados por Marín-Bernal y Navarro-Ríos (2014) en ovinos Segureños de España. Un 87,5\% de los AGRICH poseían menos de 20 ovinos, con un promedio de 13,2 por criador (Tabla 1).

Al clasificar si el sistema es comercial o de subsistencia, de acuerdo con Díaz (2013) los rebaños de los PASTHU serían comerciales por tener más de 50 ovinos y los rebaños de los AGRICH de subsistencia por tener menor número. Según Galarza (2006) las explotaciones de subsistencia son pequeñas y para consumo de los criadores, en cuanto las comerciales tienen rebaños numerosos, con énfasis en la venta al mercado.

Tabla 1. Valores promedio de ovinos, carneros y ovejas en los rebaños de los ovinocultores entrevistados en Ite, Perú

\begin{tabular}{ccccc}
\hline \multirow{2}{*}{ Resultados } & \multicolumn{2}{c}{ PASTHU } & \multicolumn{2}{c}{ AGRICH } \\
\cline { 2 - 5 } & Promedio & DS & Promedio & DS \\
\hline Ovinos & $189,0^{\mathrm{a}}$ & 15,5 & $13,2^{\mathrm{b}}$ & 8,9 \\
Valor promedio Carneros & $3,9^{\mathrm{a}}$ & 0,5 & $1,4^{\mathrm{b}}$ & 0,3 \\
Ovejas & $140,0^{\mathrm{a}}$ & 13,9 & $7,5^{\mathrm{b}}$ & 8,0 \\
\hline
\end{tabular}

PASTHU $=$ Pastores de los Humedales; AGRICH $=$ Agricultores de las chacras; DS = Desvío Estándar. Promedio de los valores absolutos obtenidos en las respuestas cuantitativas de las entrevistas; Promedios con letra diferente $(\mathrm{a}, \mathrm{b})$ no son iguales por el Test Scott-Knott al 5\%

Los PASTHU poseían en promedio 3,9 reproductores, con rebaños de dos o hasta siete carneros. Los AGRICH tenían en promedio 1,4 reproductores, siendo común encontrar apenas un carnero en las propiedades. En el caso de las hembras, el número era en promedio 140 para los PASTHU, registrando rebaños de 50 hasta 300 ovejas. Los AGRICH criaban 7,5 ovejas por propiedad, con rebaños de dos o hasta 26 hembras.

Al calcular el número de ovejas que sirve un carnero, en los Humedales fueron 31,1 por cada reproductor, número adecuado para la especie según Jainudeen et al. (2002), pero inferior al descrito en otros estudios, con 37,9 (Marín-Bernal y Navarro-Ríos, 2014) y 42,1 (González et al., 2007) ovejas por reproductor. En las chacras eran cinco hembras por cada carnero, esta baja relación puede tener efecto negativo en el mejoramiento genético, pues reduce la presión de selección (Morantes et al., 2008).

Para reconocer sus ovinos generalmente los PASTHU (75\%) y algunos AGRICH (20,8\%) usaban muescas en las orejas, con marcas específicas de cada dueño, método también encontrando por González et al. (2007) entre criadores de Ovejas Canarias. Cuando los ovinos se extraviaban, varios entrevistados sabían diferenciarlos visualmente (PASTHU 50\%; AGRICH 83,3\%), pues recordaban detalles corporales de sus animales. Esto prueba la habilidad pastoril del hombre peruano de campo, que cría rebaños de camélidos sudamericanos desde épocas pre-colombinas (Gade, 2013).

Ningún entrevistado usaba registros zootécnicos, aretes, tatuajes o collares en sus ovinos, lo que puede ser una desventaja, pues no permite la identificación individual, impidiendo el fácil manejo y acompañamiento productivo (Alencar et al., 2010). Un PASTHU y tres AGRICH llamaban a sus ovinos por nombre, costumbre también registrada en las pastoras mexicanas Tzotziles (Perezgrovas y Castro, 2000). Cuando se preguntó la edad de los ovinos, el 50\% de los PASTHU y 45,8\% de los AGRICH sabían determinarla mediante cronología 
dentaria, algo que es necesario para mejor monitoreo en cada fase de la vida de esos rumiantes (Silva et al., 2001). Muchos PASTHU (75\%) y AGRICH (83,3\%) usaba el número de partos como parámetro de edad en las ovejas.

En Ite, según el tipo de ambiente y productor fueron observados diversos sistemas de crianza ovina (Tabla 2).

Tabla 2. Sistemas de crianza de los ovinocultores entrevistados en Ite, Perú

\begin{tabular}{llcccc}
\hline \multirow{2}{*}{ Resultados } & \multicolumn{2}{c}{ PASTHU } & \multicolumn{2}{c}{ AGRICH } \\
\cline { 2 - 5 } & Pastoreo semiextensivo & $\mathrm{n} / \mathrm{N}$ & $\%$ & $\mathrm{n} / \mathrm{N}$ & $\%$ \\
\hline \multirow{2}{*}{$\begin{array}{l}\text { Sistemas } \\
\text { extractivista }\end{array}$} & $8 / 8$ & 100 & 0 & 0 \\
\cline { 2 - 6 } $\begin{array}{l}\text { de } \\
\text { crianza }\end{array}$ & Estaca y corral & 0 & 0 & $10 / 24$ & 41,7 \\
& Estaca permanente & 0 & 0 & $11 / 24$ & 45,8 \\
& Pastoreo semiextensivo & 0 & 0 & $1 / 24$ & 4,2 \\
& Estabulado & 0 & 0 & $2 / 24$ & 8,3 \\
\hline
\end{tabular}

$\overline{\text { PASTHU }=\text { Pastores de los Humedales } ; \text { AGRICH }=\text { Agricultores de las }}$ chacras

En los Humedales los ovinocultores practican un pastoreo semiextensivo extractivista, utilizando la vegetación nativa de ese ecosistema costero, con plantas como Distichlis spicata, Cynodon dactylon, Bacopa monnieri, Typha angustifólia, Scirpus californicus, entre otras (Montesinos et al., 2012). Estos PASTHU liberan sus animales del corral generalmente a las seis de la mañana y los recogen cinco de la tarde para pernoctar, con un promedio de 9,5 horas a campo. Intervalo de tiempo semejante al reportado por Galarza (2006) en ovinos criollos bolivianos, con ocho a diez horas. Este extractivismo también fue descrito por De la Barra et al. (2011) en ovinos Chilote, que consumen la vegetación litoránea del archipiélago de Chiloé y por Santos et al. (2010) en ovicaprinocultores del semiárido brasilero, que utilizan los recursos forrajeros del ecosistema caatinga. Apenas cuatro PASTHU suplementaban por la tarde sus rebaños con chala de maíz, así como otros dos criadores daban alfalfa y grama.

Estos PASTHU realizaban ocasionalmente trashumancia (Montesinos et al., 2015), para aprovechar la flora nativa de otro ecosistema llamado Lomas, el cual crece de forma temporal en los morros áridos de la costa peruana, gracias a las lluvias que trae el fenómeno de "El Niño", con especies como Alternanthera halimifolia, Lippia nodiflora y Verbena litoralis (Dillon y Rundel, 1990; Paniagua et al., 2009). Esta trashumancia fue descrita por Soto (1995), en la sociedad pastoril Aymara de los Andes bolivianos, que aprovecha la flora altoandina y realiza migraciones en busca de mejores pastos. Según los PASTHU esta vegetación de las Lomas mejora la condición corporal, crecimiento de las crías, producción láctea en las hembras y palatabilidad de la carne.

En las chacras los ovinocultores practican tres tipos de manejo. La mayoría $(87,5 \%)$ aplicaba el sistema de estaca, un AGRICH pastoreaba de manera semiextensiva y dos AGRICH estabulaban permanentemente sus rebaños (Figura 4).

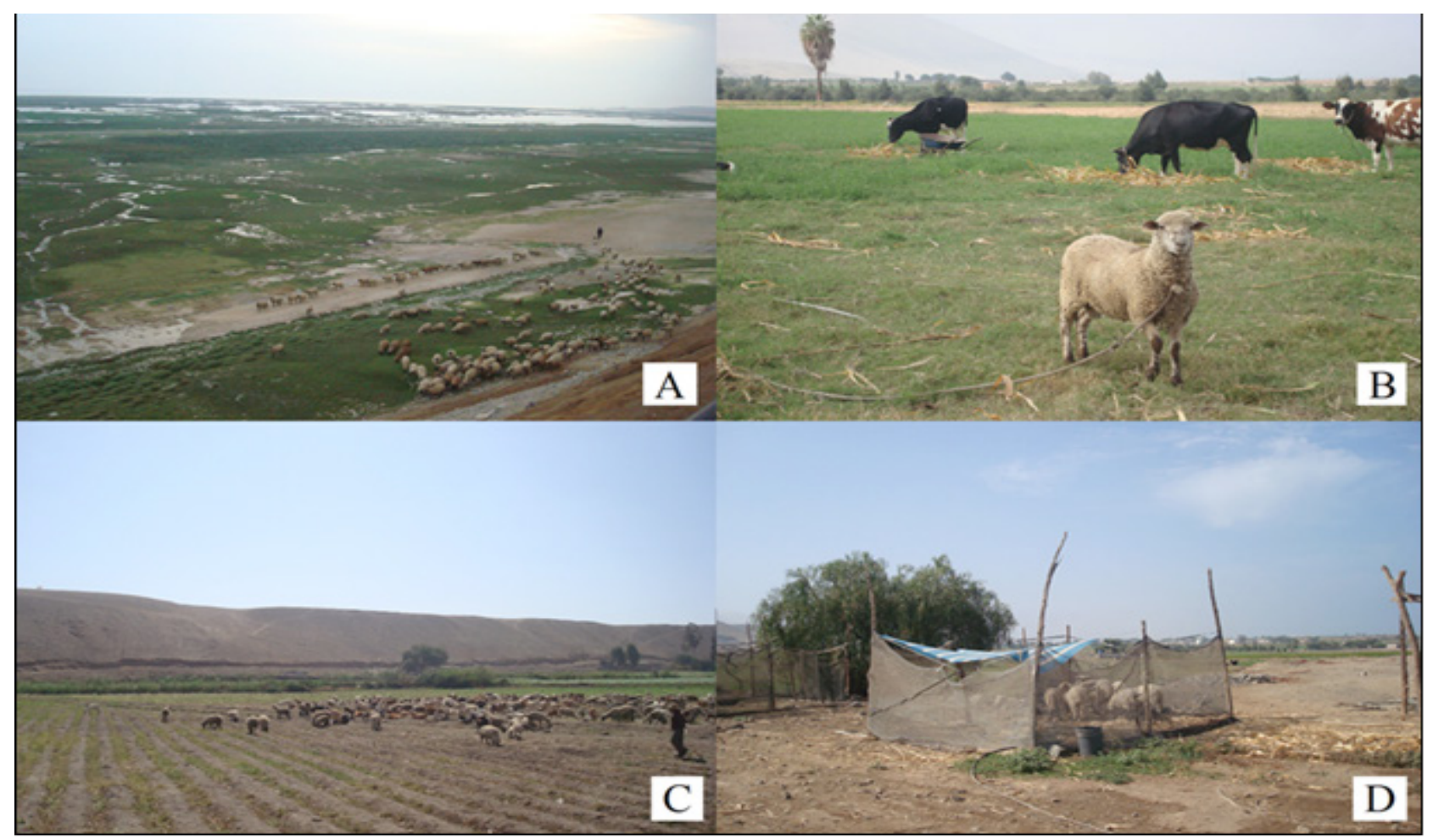

Figura 4. Pastoreo semiextensivo de vegetación nativa en los Humedales de Ite (A). Sistema de estaca con bovinos lecheros en campos de alfalfa y como suplemento chala de maíz (B). Pastoreo semiextensivo en rastrojos de cebolla (C). Rebaño estabulado en corral consumiendo silaje más chala de maíz (D) 
El manejo de estaca practicado por 21 de los $24 \mathrm{AGRICH}$ es descrito como una variante del pastoreo controlado, siendo bastante utilizado en Perú por los ganaderos (Díaz y Vilcanqui, 2013), en México por pastores Tzotzil (Perezgrovas y Castro, 2000) y en Colombia por pequeños agricultores familiares (Anzola et al., 1981). Este sistema consiste en confinar al animal en un diámetro de pastoreo, mediante una cuerda amarrada al cuello y por otro extremo sujeta a una vara metálica plantada en el suelo (Montesinos et al., 2013). Para Díaz y Vilcanqui (2013) se aprovecha mejor el pasto sin gastar en infraestructura, pero el éxito depende del encargado del rebaño, que debe cambiar la posición de la estaca con la frecuencia necesaria, para alimentar al animal y proporcionarle suficiente agua.

En Ite, bajo el manejo de estaca los AGRICH crían ovinos en forma mixta con bovinos lecheros, colocándolos en "potreros" donde se alimentan de residuos de alfalfa (Medicago sativa) o pastos más bajos (Cynodon dactylon) dejados por las vacas, y con caprinos "limpiando" los canales de regadío invadidos por grama (Distichlis spicata) o los terrenos con residuos agrícolas de ají y maíz. El mismo pastoreo mixto fue indicado por Kirchof (2000) para bovinocultores en el sur de Brasil, con lo cual no necesitarían cortar el forraje que sobró de las vacas y tendrían otro ingreso vendiendo los ovinos.

El grupo de 21 AGRICH registró dos modalidades del sistema de estaca. En la primera modalidad, 10 ovinocultores llevaban sus animales del corral para el campo a las seis de la mañana, pastoreando estaqueados hasta las cinco de la tarde, utilizando en promedio 10,8 horas, después el encargado los traía al corral para pernoctar. Esos horarios y promedio son próximos a los encontrados en los Humedales y en rebaños dominicanos (Valerio et al., 2010). En la segunda modalidad, habían 11 criadores que mantenían sus ovinos a campo estaqueados permanentemente, porque carecían de corrales. Varios AGRICH querían estabular sus animales para facilitar la alimentación, ya que a estaca es trabajoso cuando son varios individuos (Díaz y Vilcanqui, 2013).

Estos 21 rebaños eran suplementados con chala, silaje o granos de maíz, rastrojos de ají y heno de alfalfa, tanto en el pastoreo a campo como en el corral. Martínez et al. (2009) reportaron que ovinos mexicanos en época de sequía eran suplementados con granos de maíz o cebada y pastoreaban en residuos agrícolas de avena o papa. Algo que realizaban los AGRICH, estaqueando animales en terrenos donde se colectó ají o maíz.

El segundo tipo de manejo era practicado apenas por un AGRICH, que criaba ovinos y caprinos semiextensivamente, asumiendo la función de pastor por unas horas, al salir al mediodía con su rebaño en busca de rastrojos (ají o maíz) y grama en las chacras vecinas, volviendo en la tarde al corral. Este consumo de alimentos groseros confirma que los ovinos aprovechan todo tipo de vegetación (Fulcrand, 2006). Ese propietario suplementaba sus animales en el corral, con alfalfa o chala de maíz, antes del medio día que salían para pastoreo y después de las cinco de la tarde, cuando volvían para pernoctar.
El último tipo de manejo fue descrito por dos AGRICH, que estabulaban ovinos permanentemente, ofreciendo dos y tres veces al día alimentos en el comedero (alfalfa, grama, chala o silaje de maíz). Este sistema también era realizado de forma ocasional si faltaba forraje en invierno. Ovinocultores mexicanos (Martínez et al., 2009) y caprinocultores del semiárido brasilero (Costa et al., 2008) estabulaban en la época de sequía, ya que al tener pocos animales era factible ese manejo.

La mitad de los PASTHU compraban concentrado para bovinos, en lugar de algo específico para ovinos, debido a falta de disponibilidad en el comercio regional. Los PASTHU priorizaban dar concentrado a ovejas recién paridas, corderos, reproductores y borregos en etapa de engorde. Tres AGRICH lo usaban en ovejas postparto y un AGRICH cuando faltaba forraje en invierno. Los entrevistados indicaron la importancia de las hembras como base para el desarrollo del rebaño y por eso su mejor trato. Aquellos ovinocultores que no compraban concentrado manifestaron falta de dinero, costumbre y/o comederos en el corral para administrarlo. Costa et al. (2008) registraron el uso de concentrado en rebaños ovinos y caprinos del nordeste brasilero, cuando las hembras estaban en fase de lactación, o en el periodo de sequía para evitar desnutrición. Góngora et al. (2010) indicaron que en rebaños extensivos mexicanos la oferta de concentrado era principalmente para ovejas, reproductores y después las crías.

Al preguntar sobre facilidades existentes en la ovinocultura, los ocho PASTHU manifestaron tener afición y costumbre familiar para criar la especie. En segundo lugar, 75\% resaltó el extractivismo de la vegetación nativa en los Humedales, que no representa gasto económico, ya que apenas sueltan y recogen sus animales (Montesinos et al., 2012). Situación semejante a ovinos de los Andes peruanos, que consumen exclusivamente pastos naturales, dando valor a extensas áreas no aptas para agricultura (Fulcrand, 2006). Un 62,5\% de los PASTHU consideró el aprovechamiento integral del ovino y la rápida comercialización de subproductos. Martínez et al. (2009) destacaron esas dos ventajas como fortalezas, por generar dinero en efectivo. La mitad de los PASTHU reconocieron que los ovinos son de crianza simple, ciclo biológico corto, pudiendo pastorear en terrenos inaccesibles (Flores, 2008). Tres PASTHU relataron que la mano de obra es el único gasto en ese sistema extractivista, sin precisar mucho capital económico de inversión. Un PASTHU apuntó la capacidad del ovino para alimentarse de variada y poca vegetación, así como beber poca agua. Características de rusticidad también descritas por Fulcrand (2005), en ovejas criollas de los Andes peruanos.

El 75\% de los AGRICH apuntaron como facilidades de la ovinocultura, el aprovechamiento integral del animal y fácil venta de subproductos (carne, lana y estiércol). Seguidamente el 58,3\%, mencionó la costumbre y afición por criar ovinos, pues los propietarios sentían orgullo y estatus social al poseer un rebaño numeroso (Quijandría, 1985), o un reproductor de gran tamaño, "mayor que los 
carneros de los vecinos". Otro 58,3\% de los AGRICH comentó que el ovino es de crianza simple, dócil e instinto gregario, lo cual permite su manejo en conjunto (Aliaga, 2009), así como un ciclo productivo más corto que sus bovinos, pues tiene mayor eficiencia biológica, al ser más prolífico y requerir menos tiempo de gestación o beneficio (Galarza, 2006; Reis et al., 2011). La resistencia y adaptación para consumir poca agua y variada vegetación fue indicada por $45,8 \%$ de los AGRICH y la practicidad del manejo a estaca por otro $20,8 \%$, ya que es un sistema que no precisa de corral (Díaz y Vilcanqui, 2013).

Como dificultades de la actividad, la mitad de los PASTHU indicó gastos económicos por enfermedades, seguidamente dos PASTHU dijeron los cuidados en el parto y post-parto para las crías, que dependen de la habilidad materna de cada oveja (Rech et al., 2011). Varios PASTHU comentaron que los corderos requieren menos atenciones que los cabritos, pues nacen y permanecen al pie de la oveja, ya los otros tienen que ser llevados hasta la cabra para mamar calostro. Dos PASTHU criticaron la baja calidad de la vegetación nativa de ese ecosistema, sirviendo solo para mantener al animal y no para engordarlo. Una PASTHU relató que es una actividad esclavizante, por tener que visitar dos veces al día su rebaño. Fue detectado cierto conformismo en los PASTHU, pues decían "criar ovinos por criar" o "a la antigua", lo que según Leiva et al. (2011) indicaría dificultad en adoptar tecnologías, tal vez por su bajo nivel escolar.

Los AGRICH mencionaron entre dificultades de la ovinocultura, la falta de forraje en invierno $(25 \%)$ y que el ovino destruye las cosechas cuando escapa de la estaca o el corral (12,5\%). Dos AGRICH $(8,3 \%)$ reclamaron del ataque de raposas, perros de los vecinos y robos, problemas también descritos en rebaños mexicanos y brasileros (Nogueira et al., 2007; Morantes et al., 2008; Aferri y Barbosa, 2014). Dos AGRICH (8,3\%) apuntaron el gasto económico para tratar enfermedades y otros dos $(8,3 \%)$ el trabajo que da alimentar los ovinos diariamente.

En Ite fueron distinguidos tres grupos étnicos de ovinos (Figura 5), los criollos, los mestizos Hampshire Down (HD) y Black Belly (BB).

Estos criollos o "chuños", como eran llamados por los criadores, pertenecían mayoritariamente a la variedad de cara y lana blanca, siendo encontrados en todos los rebaños de los PASTHU y en la mayoría de los AGRICH (70,8\%). También existían criollos con cara y lana negra, así como cara y lana amarilla, pero esas variedades en cantidad ínfima tal vez por estar casi extintas. Fue verificado por las imágenes documentadas, que las tres variedades de ovinos locales de Ite son parecidas a las tres variedades

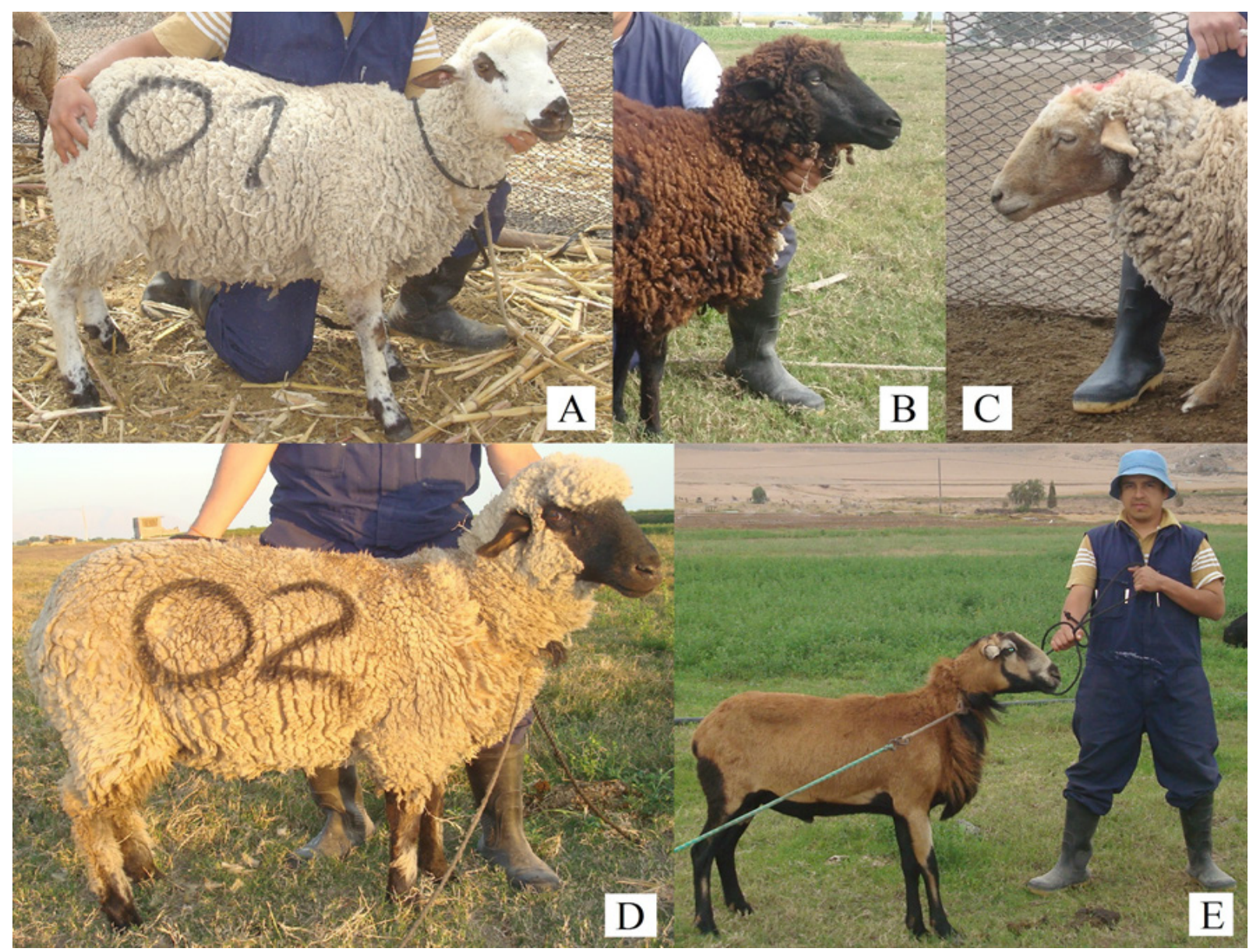

Figura 5. Ovejas criollas “cara blanca” (A), negra (B) y castaña (C). Oveja mestiza Hampshire Down (D) y carnero Black Belly (E) 
del borrego mexicano Chiapas, descritas por Pedraza et al. (1992). Probablemente porque tienen los mismos troncos ancestrales, que llegaron a Perú y México con la colonización española, entre los cuales destaca la oveja Churra.

Los mestizos HD o "cara negra", denominados así por los ovinocultores, mostraban lana blanca o algo amarillenta y biotipo cárnico (Mendives, 2007), siendo criados por todos los PASTHU y AGRICH, generalmente para substituir sus ovinos criollos. Estos mestizos descienden del HD importado en la década de 1980, para la región costera peruana (Flores, 2008), y de aquel donado en 1996, por el Ministerio de Agricultura del Perú, para diferentes lugares de la región Tacna (MINAG-DRAT, 2004). Tres AGRICH también criaban ovinos Black Belly (BB) y sus mestizos, raza tropical introducida en 1984 a la selva amazónica peruana (CEDAP, 1997) y después al ambiente costero (Bañados et al., 2006), como aconteció en estos últimos años en Ite.

En Perú, generalmente las razas exóticas están presentes debido a donaciones gubernamentales, para fomentar el cruzamiento absorbente y "mejorar" la productividad de los rebaños criollos. En Ite, existían cruzamientos entre los tres grupos étnicos encontrados, situación también descrita en rebaños de pequeñas comunidades en la sierra central peruana (Flores et al., 2003), donde el empadre de ovejas criollas y mestizas con machos Corriedale, Junín y HD es habitual. Esas cruzas afectan la genética criolla y su sobrevivencia, pudiendo extinguirla, lo que sería una gran pérdida, pues desaparecerían informaciones contenidas en su estructura genética, desarrolladas a través de los siglos por selección natural (Mariante y Egito, 2002; López et al., 2013).

La rusticidad y adaptación fueron las características más destacadas de los ovinos criollos, según $100 \%$ de los PASTHU y 70,6\% de los AGRICH que criaban esos ejemplares, sobre todo por su resistencia al clima adverso, vermes gastrointestinales y enfermedades respiratorias o podales, características igualmente reportadas en ovinos criollos del archipiélago de Chiloé (De la Barra et al., 2011). Los rebaños de los PASTHU estaban adaptados a tierras pantanosas (Montesinos et al., 2012), con alta carga parasitaria, registrándose relatos que eran resistentes a la Fasciola hepática, algo que también destacó Fulcrand (2005) en ovinos criollos de la sierra peruana.

Tanto los PASTHU (50\%) como AGRICH (58,8\%) resaltaron que el ovino criollo come poco por ser pequeño (Stemmer et al., 2010) y aprovecha diferentes tipos de vegetación, como pastos groseros (Fulcrand, 2005), representando bajos costos de mantenimiento para el criador (Aliaga, 2009). Tres PASTHU y dos AGRICH dijeron que los ovinos criollos poseían carne sabrosa y magra, diferente a la carne grasosa de los mestizos HD (Fulcrand, 2005; Gonzáles, 2008). La carne de los criollos de los Humedales es menos grasosa, porque están sometidos a grandes caminatas en busca de alimento (Montesinos et al., 2012). Estas características dan valor agregado a la carne, justificando la crianza de "ovinos criollos puros" (Stemmer et al., 2010).

Un PASTHU mencionó la notable habilidad materna de las ovejas criollas, como también indicaron Gómez y Gómez (2009), en ovejas locales de la sierra peruana. El mismo productor destacó el mayor líbido de los carneros criollos, en comparación a mestizos HD, ya que muchas veces hasta enfermos montaban las hembras ante la mirada pasiva de los "cara negra", que eran pesados y lentos para cópula. Ventajas reproductivas descritas en el carnero Chiapas (Pimentel et al., 2005), siendo interesante tipificar la reproducción de los machos criollos en Ite, para formar núcleos de conservación y mejoramiento a disposición de los criadores. Dos AGRICH afirmaron que algunos ovinos criollos eran grandes y pesados, tal vez por tratarse de un linaje cárnico, variabilidad criolla observada en otras regiones del país (Fulcrand, 2005), por lo cual es necesario el genotipo de estos rebaños en Ite.

El lado negativo de los criollos, reconocido por $75 \%$ de los PASTHU y 58,8\% de los AGRICH era su poco peso y tamaño, así como ser tardíos en crecimiento según dos PASTHU y tres AGRICH. Esto debido a formas de crianza tradicional, que mantienen la producción estática (Alencastre y Gómez, 2005). Los ovinos criollos de Ite no poseen aptitud especializada, siendo criados apenas para carne ya que las ovejas no se ordeñan, como también fue reportado en rebaños criollos Chilote (De la Barra et al., 2011).

En el caso de los mestizos HD, todos los PASTHU y el 95,8\% de los AGRICH destacaron que eran animales cárnicos, pesados y con mayor tamaño que los criollos. También el 62,5\% de los AGRICH relataron precocidad para ganar peso, ya que a los tres meses de edad alcanzaban cerca de 15 kilos de peso vivo, en comparación a los criollos que eran tardíos y con peso inferior, lo que podía deberse a factores raciales y sobretodo manejo (Bañados et al., 2006). Tres AGRICH valorizaban la habilidad del ovino mestizo para comer variada vegetación, en comparación a sus bovinos lecheros (Mendives, 2007). Un AGRICH apuntó la prolificidad de las ovejas BB, teniendo hasta tres crías por parto, particularidad de esa raza deslanada tropical (Gonzáles, 2008).

La desventaja más notoria del ovino mestizo según PASTHU (62,5\%) y AGRICH (45,8\%) es la susceptibilidad a enfermedades (fasciolosis, sarna y neumonía). Dos PASTHU y un AGRICH dijeron eran exigentes en cantidad de forraje, características ya descritas por Browman (1987) y Fulcrand (2005). Un PASTHU apuntó que los machos mestizos son tardíos sexualmente y un AGRICH describió a los BB como ovinos osudos y descarnados, esto debido a su biotipo longilíneo (Bañados et al., 2006).

Algunas ovejas criollas y mestizas tenían glándulas mamarias diferenciadas, en relación al resto de su rebaño. Esto puede indicar que ciertos grupos y/o linajes poseen aptitud lechera, lo cual podría ser otra alternativa de producción en Ite si es explorada, mediante la búsqueda y selección de este tipo de ovejas, que servirían para formar 
un rebaño matriz, donde serían mejoradas y sus crías repartidas entre los productores.

\section{Conclusiones}

Los dos tipos de ovinocultores familiares encontrados fundamentalmente se dedican a la producción de carne. Para los pastores de los Humedales es una crianza comercial y extractivista, considerada su principal actividad económica; y para los agricultores de las chacras, es una actividad secundaria y de subsistencia, destacando el autoconsumo de los animales. Se observó diferentes sistemas de producción, basados generalmente en el pastoreo. También fue registrada una preocupante reducción de los ovinos criollos, debido a cruzamientos absorbentes con mestizos Hampshire Down, buscando mejorar el peso de los animales. En Ite, la producción ovina es de forma tradicional, subsistiendo porque la carne es apreciada en la región y la crianza una costumbre familiar. La asistencia técnica y extensión rural son inadecuadas, lo que afecta de forma negativa a toda la cadena productiva ovina. Por eso es necesario un mayor apoyo gubernamental, para aplicar tecnologías que permitan una ovinocultura sustentable en esta región sur del Perú.

\section{Literatura citada}

Aferri, G. y Barbosa, C.M.P. 2014. Utilização de pasto na produção de ovinos. Pesquisa \& Tecnologia, 11 (2).

Alencar, S.P.; Mota, R.A.; Coelho, M.C.O.C.; Nascimento, A.S.; Abreu, S.R.O. y Castro, R.S. 2010. Perfil sanitário dos rebanhos caprinos e ovinos no sertão de Pernambuco. Ciência Animal Brasileira 11 (1): 131140.

Alencastre, R.D. y Gómez, N.U. 2005. Comportamiento reproductivo del ovino criollo en el altiplano peruano. Archivos de Zootecnia 54 (206-207): 541-544.

Aliaga, J.L. 2009. Posibilidades del desarrollo de la crianza ovina en el Perú. En: Foro Regional sobre Ovinos Criollos, $3^{\circ}$, Cusco, Perú, 2009.

Altafin, I.; Pinheiro, M.E.F.; Valone, G.V. y Gregolin, A.C. 2011. Produção familiar de leite no Brasil: um estudo sobre os assentamentos de reforma agrária no Município de Unaí (MG). Revista UNI 1 (1): 31-49.

Anzola, H.V.; Naranjo, A.Q.; Ferguson, J. y Montes, M. 1981. Sistemas de producción ovina y su nivel tecnológico en la zona central de Colombia. ICA, Bogotá, Colombia. 36 pp.

Arnhold, E. 2013. Package in the R environment for analysis of variance and complementary analyses. Brazilian Journal of Veterinary Research and Animal Science 50 (6): 488-492.

Avilés, D.F.; Martínez, A.M.; Landi, V. y Delgado, J.V. 2014. El cuy (Cavia porcellus): un recurso andino de interés agroalimentario. Animal Genetic Resources 55: 87-91.

Bañados, A.M.; Aliaga, J.G. y Chauca, L.F. 2006.
Evaluación comparativa del crecimiento de ovinos Barriga Negra puros y cruzados con criollos de Piura bajo sistema intensivo en la costa central. Anales cientificos 66: 174-189.

Bedotti, D.; Gómez Castro, A.G.; Sánchez Rodríguez, M.; García Martínez, A. y Martos Peinado, J. 2005. Aspectos sociológicos de los sistemas de producción caprina en el oeste pampeano (Argentina). Archivos de Zootecnia 54 (208): 599-608.

Browman, D.L. 1987. Agro-pastoril risk management in the Central Andes. Research in Economic Anthropology 8: $171-200$.

CEDAP (Centro de Estudios y Desarrollo Agrario del Perú). 1997. Proyecto sistemas de producción de crianzas familiares. Recuperado de:https://idl-bnc-idrc.dspacedirect.org/ bitstream/handle/10625/35249/108039-v1. pdf? sequence $=1 \&$ is Allowed $=y$

Costa, R.G.; Almeida, C.C.; Pimenta Filho, E.C.; Holanda Junior, E.V. y Santos, N.M. 2008. Caracterização do sistema de produção caprino e ovino na região semiárida do estado da Paraíba, Brasil. Archivos de Zootecnia 57 (218): 195-205.

Daskolopoulou, I. y Petrou, A. 2002. Utilising a farm typology to identify potential adopters of alternative farming activities in Greek agriculture. Journal of Rural Studies 18: 95-103.

De la Barra, R.; Carvajal, A.; Uribe, H.; Martínez, M.E.; Gonzalo, C.; Arranz, J. y San Primitivo, F. 2011. El ovino criollo Chilote y su potencial productivo. Animal Genetic Resources 48: 93-99.

De la Rosa, S.; Revidatti, M.A.; Orga, A.; Tejerina, E.; Capello, S. y Pilotti, P. 2014. Manejo tradicional de las majadas de ovejas criollas del oeste formoseño. Actas Iberoamericanas de Conservación Animal 4: 305-307.

Díaz, R.I.R. 2007. Sector ovinos en el Perú con perspectivas al 2015. En: Congreso de Especialistas en Pequeños Rumiantes y Camélidos Sudamericanos, $5^{\circ}$, Mendoza, Argentina, 2007.

Díaz, R.I.R. 2013. Cadena productiva de ovinos. MINAGRI, Lima, Perú. 53 pp.

Díaz, R.I.R. y Vilcanqui, H.M. 2013. Manual de ovinos y las buenas prácticas. MINAGRI, Lima, Perú. 95 pp.

Dillon, M.O. y Rundel, P.W. 1990. The botanical response of the Atacama and Peruvian desert floras to the 198283 El Niño event. En: Glynn, P.W. Global ecological consequences of the 1982-83 El Niño-Southern Oscillation. Elsevier, Amsterdam, Holanda. pp. 487504.

Escárzaga, F.; Abanto, J.L. y Chamorro, A.G. 2002. Migración, guerra interna e identidad andina en Perú. Política y Cultura, 18: 277-298.

Farias, J.L.S.; Araújo, M.R.A.; Lima, A.R.; Alves, F.S.F.; Oliveira, L.S. y Souza, H.A. 2014. Análise socioeconômica de produtores familiares de caprinos 
e ovinos no semiárido cearense, Brasil. Archivos de Zootecnia, 63 (241): 13-24.

Flores, E.R.; Cruz, J.Á. y López, M. 2003. Manejo y uso de los recursos genéticos en los sistemas de producción ovina de los Andes Centrales del Perú. En: Uso y manejo de los recursos genéticos de rumiantes menores en sistemas ganaderos tradicionales en países de Sudamérica; 1ㅜ, Bariloche, Argentina, 2003.

Flores, E.R.; Cruz, J.A. y López, M. 2007. Management of sheep genetic resources in the central Andes of Peru. En: Tempelman, K., Cardellino, R.A. People and animals. Traditional livestock keepers: guardians of domestic animal diversity. FAO, Roma, Italia. pp. 47-57.

Flores, D.S. 2008. Crianza de ovinos. http://www.articulo. org/articulo/3625/crianza_de_ovinos_i.html

Fulcrand, B.T. 2005. Melhorando o desempenho de raças locais de ovelhas. Agriculturas, 2 (4): 16-19.

Fulcrand, B.T. 2006. Melhoramento e desempenho de raças locais de pequenos ruminantes experiência no Peru. En: Semana da Caprinocultura e Ovinocultura Brasileiras, $5^{\circ}$, Campo Grande, Brasil, 2006.

Gade, D.W. 2013. Llamas and alpacas as "Sheep" in the colonial Andes: Zoogeography meets eurocentrism. Journal of Latin American Geography, 12 (2): 221243.

Galarza, A.B. 2006. Importancia de los ovinos criollos para los pequeños productores en alturas y cabeceras de valle del departamento de Cochabamba. En: Taller Iberoamericano sobre el Uso Sustentable de las Razas Caprinas y Ovinas locales en Áreas Marginales, $1^{\circ}$, Cochabamba, Bolivia, 2006.

Gómez, N.C.U. y Gómez, J.W.U. 2009. Importancia del recurso ovino peruano en el desarrollo rural sostenible. En: Delgado, J.V., Nogales, S. Biodiversidad ovina Iberoamericana. Caracterización y uso sustentable. UCO, Córdoba, España. pp. 387-403.

Góngora, R.D.P.; Góngora, S.F.G.; Magaña, M.A.M. y Lara, P.E.L. 2010. Caracterización técnica y socioeconómica de la producción ovina en el estado de Yucatán, México. Agronomía Mesoamericana 21 (1): 131-144.

Gonzáles, E.C.D. 2008. Producción de ganado ovino en Perú. http://www.monografias.com/trabajos58/ produccion-ovina-peru/produccion-ovina-peru.shtml

González, C.; Fernández, G. y Barba, C. 2007. Aproximación a la caracterización de los sistemas de explotación de la oveja Canaria en Gran Canaria. Archivos de Zootecnia 56 (1): 759-764.

Hernández, L.M.R. 2012. La capacitación de la mujer rural, una necesidad inaplazable para el fortalecimiento y desarrollo del sector agropecuario. Agroentorno, noviembre, 5-8.

Holanda Junior, E.V.; Oliveira, C.A.V.; Silva, P.C.G.; Guedes, C.T.S.; Araújo, G.G.L.; Silva, C.N. y
Cezimbra, C.M. 2004. Tipologia e estrutura da renda de caprino-ovinocultores de base familiar no sertão baiano do São Francisco. En: Encontro da Sociedade Brasileira de Sistemas de Produção, $6^{\circ}$, Aracajú, Brasil, 2004.

Holanda Junior, E.V. 2005. Cabrito ecológico da caatinga um projeto em movimento. Agriculturas 2 (4): 14-15.

INEI (Instituto Nacional de Estadística e Informática). 2013. Resultados definitivos IV Censo Nacional Agropecuario 2012. MINAGRI, Lima, Perú. 62 pp.

Jainudeen, M.R.; Wahid, H. y Hafez, E.S.E. 2002. Ovejas y cabras. En: Hafez, E.S.S.E., Hafez, B. Reproducción e inseminación artificial en animales. 7a ed. McGraw Hill - Interamericana, DF, México. pp. 177-187.

Kirchof, B. 2000. Produção de leite a pasto / Pastoreio rotativo. Agroecologia e Desenvolvimento Rural Sustentável, 4.

Leiva, Y.; Aguilera, R.; Meyer, J.; Avilez, J. y Neumann, J. 2011. Eficiencia del uso de la inseminación artificial vía intracervical con semen fresco en ovejas de productores Mapuche de la comuna Perquenco, Región de la Araucanía, Chile. Spermova 1 (1): 125-126.

López, P.E.; Pro, M.A.; Cuca, G.J.M. y Pérez, H.P. 2013. Ganadería de Traspatio en México y seguridad alimentaria situación actual y perspectivas. Agroentorno, mayo, 38-40.

Mariante, A.S. and Egito, A.A. 2002. Animal genetic resources in Brazil: Result of five centuries of natural selection. Theriogenology 57: 223-235.

Marín-Bernal, A.M. y Navarro-Ríos, M.J. 2014. Análisis y diagnóstico de los sistemas de producción ovina en el sureste español. Archivos de Zootecnia 63 (243): 519529.

Martínez, I.V.; Vargas, S.L.; Zaragoza, J.L.R.; González, A.B.; Calderón, F.S.; Rojas, J.Á. y Casiano, M.A.V. 2009. Tipología de explotaciones ovinas en la sierra norte del estado de Puebla. Técnica Pecuaria en México 47 (4): 357-369.

MDI (Municipalidad Distrital de Ite). 2014a. Censo poblacional de rumiantes menores del distrito de Ite. Proyecto Mejoramiento de capacidades en buenas prácticas pecuarias, para el tratamiento del ganado ovino.

MDI (Municipalidad Distrital de Ite). 2014b. Plan de desarrollo concertado al 2021. http://www.muniite. gob.pe/transparencia/planiamiento-y-organizacion/ PDC-CAPITULO-I-II-III.pdf

Mendives, J.A.A. 2007. Importancia de los ovinos tropicales introducidos al país: características productivas y reproductivas. Archivos Latinoamericanos de Producción Animal, 15 (spl. 1): 310-315.

MINAG-DRAT (Ministerio de Agricultura - Dirección Regional de Agricultura Tacna). 2004. Diagnóstico Agrario 2004 Región Tacna - Ovinos. pp. 445-447.

MINAG (Ministerio de Agricultura). 2007. Estudio de 
delimitación del distrito de Riego Locumba Sama. https:/pt.scribd.com/document/315813310/Estudiode-Delimitacion-Del-Distrito-de-Riego-LocumbaSama

Montesinos, I.S.; Silva, M.C.; Lopes, F.B.; Fioravanti, M.C.S.; McManus, C.M. y Sereno, J.R.B. 2012. Caracterização fenotípica de ovelhas dos Humedales de Ite, sul do Peru: dados preliminares. Archivos de Zootecnia 61 (236): 505-515.

Montesinos, I.S., Silva, M.C., Silva, B.A. e Sereno, J.R.B. 2013. Reprodução assistida em caprinos do litoral sul do Peru: relato de caso. En: Congresso Brasileiro de Reprodução Animal, 20, Uberlândia, Brasil, 2013.

Montesinos, I.S.; Catachura, A.; Sánchez, J.; Franco, J.L.; Arnhold, E.; McManus, C.; Fioravanti, M.C.S. y Sereno, J.R.B. 2015. Caracterización de ovinos en el litoral sur del Perú. Animal Genetic Resources 56: 5562.

Morantes, M.; Rondón, Z.; Colmenares, O.; Ríos, L.A. y Zambrano, C. 2008. Análisis descriptivo de los sistemas de producción con ovinos en el municipio de San Genaro de Boconoito (Estado Portuguesa, Venezuela). Revista Cientifica 18 (5): 556-561.

Nogueira, A.H.C.; Curci, V.C.L.M.; Ferrari, C.I.L. y Cardoso, T.C. 2007. Aspectos epidemiológicos da ovinocultura na região de Araçatuba - dados preliminares. Biológico 68 (1): 17.

Nuncio-Ochoa, G.; Nahed, J.T.; Díaz, B.H.; Escobedo, F.A. y Salvatierra, E.B.I. 2001. Caracterización de los sistemas de producción ovina en el estado de Tabasco. Agrociencia 35 (4): 469-477.

Paniagua, H.; Ignacio, J. y Silva, L. 2009. Evaluación de Carica candicans y Weberbaurella brongniartioides del ecosistema Lomas del desierto costero de la región Tacna. http://siar.regiontacna.gob.pe/admDocumento. php? accion $=$ bajar\&docadjunto $=178$

Pedraza, P.; Peralta, M. y Perezgrovas, R. 1992. El Borrego Chiapas: una raza local mexicana de origen español. Archivos de Zootecnia 41 (154): 355-362.

Peña, S.; Sacchero, D.; Maurino, J.; López, G.A.; Abbiati, N.N.; Género, E.R. y Martínez, R.D. 2016. Caracterización de la lana de ovejas Criollas argentinas en cuatro ambientes diferentes. Archivos de Zootecnia 65 (249): 13-19.

Perezgrovas, R.G. y Castro, H.G. 2000. El borrego Chiapas y el sistema tradicional de manejo de ovinos entre las pastoras tzotziles. Archivos de Zootecnia 49 (187): 391-403.

Pimentel, J.G.; Perezgrovas, R.G.; Zaragoza, L.M. y Rodríguez, G.G. 2005. Caracterización reproductiva integral del morueco en el ganado lanar de Chiapas. Archivos de Zootecnia 54 (206-207): 557-564.

Quijandría, B. 1985. Recientes avances en el Mejoramiento Genético de ovinos: Resultados del programa de Rumiantes Menores. Reportes Técnicos INIPA, 53:
$1-38$.

R Development Core Team (R): A language and environment for estatistical computing. 2011. Viena: R Foundation for Statistical Computing. http://www. Rproject.org

Rech, C.L.S.; Tarouco, A.K.; Fischer, V.; Meira, N.A.; Macêdo, J.F.; Lima, T.L. e Aita, M.F. 2011. Temperamento e comportamento materno ovino. Revista Brasileira de Reprodução Animal, 35 (3): 327340.

Reis, F.A.; Costa, J.A.A. e Gonzalez, C.I.M. 2011. Viabilidade técnica da criação de ovinos no Cerrado. En: Simpósio Sulmatogrossense de Produção Animal, $1^{\circ}$, Campo Grande, Brasil, 2011.

Santillana, M.E. 2012. La revolución silenciosa: boom económico en zonas rurales. Perú Económico, Lima, mayo, 14-16.

Santos, S.A.; Juliano, R.S.; Paiva, S.R.; Araújo, M.T.B.D. e Berselli, C. 2010. Descrição de sistemas de criação tradicionais de ovinos da Nhecolândia, Pantanal, MS. Circular Técnica, 94.

Santos, T.C.P.; Figueiredo, S.C.; Beltrão, R.A.M.S. eAlfaro, C.E.P. 2012. Aspectos gerenciais e infraestrutura em criações de caprinos e ovinos na região semiárida da Paraíba. En: Congresso Norte Nordeste de Pesquisa e Inovação, 7, Palmas, Brasil, 2012.

Segundo, P.S.; Diniz Filho, E.T.; Maracajá, V.P.B.B.; Maracajá, P.B. y Pereira, T.F.C. 2006. Agricultura familiar: um estudo sobre a juventude rural no Município de Serra do Mel - RN. Revista Verde de Agroecologia e Desenvolvimento Rural Sustentável, 1 (1): 54-56.

Silva, J.V.; Ribeiro, M.N.; Silva, L.P.G.; Pimenta Filho, E.C. y Vilar Filho, A.C. 2001. Cronologia dentária de caprinos mestiços e naturalizados criados no semiárido paraibano. Agropecuária Técnica, 22 (1-2): 45-51.

Soto, G. 1995. Ganadería andina y la sociedad pastoril aymara. En: Genin, D.; Picht, H.; Lizarazu, R.; Rodríguez, T. Wayra Pampa un sistema pastoril camélidos - ovinos del altiplano árido boliviano. IBTA, La Paz, Bolivia. pp. 27-34.

Sponenberg, D.P. and Taylor, C. 2009. Navajo-Churro sheep and wool in the United States. Animal Genetic Resources 45: 99-105.

Stemmer, A.; Galarza, A.; Fuentes, S. y Torrez, O. 2010. Importancia en la crianza familiar de ovinos criollos en Cochabamba, Bolivia. Leisa 26 (1): 31-32.

Valerio, D.; García, A.; Acero, R.; Perea, J.; Tapia, M. y Romero, M. 2010. Caracterización estructural del sistema ovino-caprino de la región noroeste de República Dominicana. Archivos de Zootecnia 59 (227): 333-343.

Vizcarra, J.K.R. 2008. Los Humedales de Ite: un potencial ecoturístico. https://pt.scribd.com/ document/196692677/Manual-Guia-Humedales-Ite. 